\title{
A importância do apoio social em pacientes coronarianos
}

\author{
Marcela Abreu-Rodrigues \\ Eliane Maria Fleury Seidl \\ Universidade de Brasília, Brasília-DF, Brasil
}

\begin{abstract}
Resumo: O presente estudo descreve a relação entre apoio social e doença coronariana tendo por base artigos empíricos ou de revisão publicados. A revisão bibliográfica foi realizada por meio de buscas eletrônicas nas bases de dados do PsycINFO, MedLine e SciELO. Após aplicação dos critérios de exclusão, 23 artigos sobre suporte social e doença coronariana foram selecionados, além de estudos clássicos sobre o tema. Conclui-se que há evidências da relação positiva entre disponibilidade de suporte social e melhora da saúde do paciente coronariano. Buscou-se caracterizar, ainda, algumas variáveis responsáveis por essa relação, uma vez que o suporte social tem se mostrado relevante para promover a adesão ao tratamento. Esses resultados justificam a importância do fornecimento de orientação ao cuidador sobre a saúde do paciente, assim como sobre as formas de prover apoio.
\end{abstract}

Palavras-chave: Doença das coronárias. Apoio social. Cuidadores.

\section{The importance of social support in coronary patients}

\begin{abstract}
This study describes the relationship between social support and coronary heart disease based on empiric or review published articles. The study bases on computerized search strategy of PsycINFO and MedLine and SciELO. Not only classic studies, but also 23 articles related to social support and coronary heart disease were selected after applying exclusion criteria. Evidences about the positive relation between availability of social support and better health outcomes of coronary patients, are shown. Variables responsible for this linkage are described, once social support has been shown relevant for treatment adherence. These circumstances justify the importance of giving instructions to the caregiver concerning patient's health as well, as how they can provide support.
\end{abstract}

Keywords: Coronary disease. Social support. Caregivers.

\section{La importancia del apoyo social en pacientes coronarios}

Resumen: Este estudio describe la relación entre apoyo social y enfermedad coronaria basada en los artículos empíricos o revisiones publicadas. La revisión bibliográfica se realizó a través de búsquedas electrónicas en bases de datos de PsycINFO, MedLine y SciELO. Después de la aplicación de los criterios de exclusión, 23 artículos sobre el apoyo social y enfermedad coronaria fueron seleccionados, así como estudios clásicos sobre el tema. De ello se desprende que hay pruebas de la relación positiva entre la disponibilidad de apoyo social y la mejora de la salud del paciente coronario. El objetivo fue caracterizar además, algunas de las variables responsables de esa relación debido a que el apoyo social ha demostrado ser importante para fomentar la adherencia al tratamiento. Estos resultados justifican la importancia de proporcionar orientación a las personas que cuidan de la salud del paciente, así como sobre la forma de prestar apoyo.

Palabras clave: Enfermedad coronaria. Apoyo social. Cuidadores. 


\section{Introdução}

Graças aos avanços da medicina, em especial nas últimas décadas, e às estratégias de saúde pública, as doenças que hoje afligem boa parte da humanidade são bem diferentes das que eram comuns em passado recente. Atualmente, as doenças mais prevalentes que acometem as pessoas são aquelas caracterizadas por curso lento e prolongado, tais como doenças cardiovasculares, câncer e derrames. Segundo Martire e Schulz (2007), poucas circunstâncias na vida adulta são tão estressantes como o diagnóstico de uma doença crônica, e a família exerce um papel importante no ajustamento psicológico e na ajuda quanto ao manejo dos sintomas dessas doenças. As autoras afirmam que relações sociais próximas afetam positivamente os sistemas biológicos, comportamentos de saúde e o bem-estar psicológico dos indivíduos. Assim, o envolvimento de outras pessoas no cuidado e apoio para o manejo de conseqüências adversas da enfermidade parece favorecer a adaptação e o ajustamento do paciente à doença crônica.

Segundo a World Health Organization (s.d.), em 2007, as doenças isquêmicas do coração mantiveram sua posição como a principal causa de morte no mundo, representando cerca de $20 \%$ do total de óbitos. De acordo com o Ministério da Saúde, morreram mais de 890 mil pessoas no ano de 2006, cerca de 290 mil devido a doenças do aparelho circulatório, sendo que mais de 85 mil mortes foram em decorrência de doenças isquêmicas do coração (Brasil, 2006). Assim, as síndromes coronarianas, como angina instável ou infarto agudo do miocárdio, são responsáveis por um grande número de hospitalizações no Brasil e no mundo. Boutin-Foster (2005a) afirma que a identificação e eliminação de fatores de risco que contribuem para a doença arterial coronariana tem sido uma grande preocupação para a saúde pública, gerando interesse de pesquisa.

A doença arterial coronariana é um distúrbio no qual depósitos de gordura se acumulam nas células que revestem a parede de uma artéria coronária e, conseqüentemente, obstruem o fluxo sanguiíneo. Os depósitos de gordura formam-se gradualmente e se desenvolvem nos grandes ramos das duas artérias coronárias principais, as quais circundam o coração e provêem sangue ao mesmo (Straub, 2005). Essa condição provoca o entupimento das artérias do coração e, em decorrência, o sofrimento do músculo cardíaco. Existem duas condições representativas da doença coronariana: a angina instável e o infarto agudo do miocárdio. A angina é uma condição de dor extrema no peito causada por restrição no suprimento de sangue para o coração que ocorre, em geral, durante momentos de esforço incomum. O infarto do miocárdio, popularmente conhecido como ataque do coração, resulta na morte do tecido cardíaco em resposta à interrupção do suprimento de sangue para o miocárdio. A sintomatologia típica - a dor retroesternal denominada angina pectoris - pode surgir abruptamente ou de forma insidiosa e é aliviada com o uso de medicação específica (Mansur e cols., 2004).

A etiologia multifatorial da doença coronariana é amplamente reconhecida. Os fatores que alteram sua história natural, isto é, as circunstâncias que aumentam as chances de um indivíduo adquirir a doença ou agravá-la, os chamados fatores de risco, classificam-se na literatura em duas modalidades. Aqueles em relação aos quais é possível intervir e modificar: pressão arterial elevada, aumento do colesterol, hábito de fumar, falta de atividade física, obesidade, diabetes e fatores denominados psicossociais, incluindo o estresse emocional. A outra modalidade abarca os fatores de risco potencialmente não modificáveis: hereditariedade, sexo (considerando a predisposição maior em homens dependendo da faixa etária) e idade, em função da tendência de aparecimento da enfermidade em faixas etárias superiores a 45 anos (Gianotti, 2002).

A ocorrência do quadro coronariano é marcada, muitas vezes, por medo, ansiedade e incertezas com relação ao retorno às atividades diárias (Boutin-Foster, 2005a). Sob tais circunstâncias, o apoio social é geralmente acionado e mobilizado como recurso para ajudar pacientes a enfrentar sua condição de saúde e as consequiências adversas da mesma. Segundo Straub (2005), lidar com eventos estressantes, como o advento de uma doença crônica, é especialmente difícil quando o indivíduo se sente isolado e/ou rejeitado socialmente. Assim, estudos apontam que suporte social adequado pode diminuir o risco de agravamento de tais doenças ao promover comportamentos relevantes para o seu manejo e controle. 
Neste estudo, optou-se por usar os termos suporte social e apoio social de modo alternado tendo em vista que a literatura científica brasileira, em especial da área psicológica, parece ter incorporado ambos como sinônimos. Na SciELO, por exemplo, foram encontrados cinco artigos com o termo suporte social e 30 artigos com o termo apoio social. Ressalta-se que todos os estudos sobre suporte social são da área psicológica e grande parte das pesquisas que utilizam o termo apoio social são da área de saúde pública, epidemologia e medicina. Além disso, confirmou-se que ambos os termos são usados com o mesmo significado em diferentes estudos (Araújo, Freire, Padilha, \& Baldisseroto, 2006; Beltrão, Vasconcelos, Pontes, \& Albuquerque, 2007; Cupertino e cols., 2006; Matsukwa, Marturano, \& Oishi, 2002; Pinto, Garcia, Bocchi, \& Carvalhaes, 2006; Seidl \& Troccóli, 2006).

O presente estudo tem por objetivo analisar a relação entre suporte social e doença coronariana tendo por base artigos empíricos ou de revisão. $\mathrm{O}$ material foi selecionado por meio de buscas eletrônicas nas bases de dados MedLine e PsycINFO para artigos estrangeiros, e SciELO para os nacionais, publicados entre 1999 e 2007.

\section{Método}

Os descritores usados na busca eletrônica foram, em língua inglesa, social support e coronary disease e suporte social e doença das coronárias para a busca em língua portuguesa.

Foram encontradas 244 referências na base de dados MedLine e 74 estudos no PsycINFO, havendo sobreposição de vários trabalhos. Foram excluídos os artigos que pesquisaram crianças e adolescentes, os que versavam sobre outras enfermidades cardíacas, além de dissertações e teses. Foram selecionados os estudos que continham os descritores em seu resumo e se referiam exclusivamente a pacientes adultos com diagnóstico de doença coronariana. A presente revisão incluiu apenas artigos internacionais, de língua inglesa, visto que nenhuma publicação nacional sobre o tema foi encontrada na base de dados SciELO.

Por fim, 23 referências foram selecionadas para essa revisão. Vale ressaltar que, além desses trabalhos, alguns capítulos de livros, textos clássicos e ar- tigos referentes ao assunto foram incluídos para delimitação da epidemiologia do agravo em foco e definição do conceito de suporte social.

Após leitura e análise dos estudos selecionados, foram identificadas categorias para o estabelecimento da relação entre suporte social e doença coronariana. Para isso, conceituou-se o termo suporte social e, em seguida, sua relação com a morbidade e mortalidade de pessoas com coronariopatias. Destacou-se o papel do apoio social na adesão ao tratamento desses pacientes e, por fim, aspectos referentes à relação entre adequação e satisfação do suporte social, e progressão da doença coronariana foram discutidos sugerindo a importância de orientação aos cuidadores.

\section{Suporte social: conceituação e classificação}

Os relacionamentos sociais exercem um papel fundamental na vida do indivíduo cumprindo importantes funções nas dimensões social, psicológica e comportamental. Estudos indicam que tanto a qualidade quanto a quantidade de relacionamentos sociais parecem afetar de forma significativa a morbidade e a mortalidade (Uchino, Cacioppo, \& Kiecolt-Glaser, 1996).

Suporte social pode ser definido, segundo Cobb (1976, p. 300), como "a informação que leva o indivíduo a acreditar que ele é cuidado, amado, estimado e que pertence a uma rede social com obrigações mútuas". O estudo de Cobb trouxe a primeira definição de suporte social aplicado ao contexto da saúde, apresentando evidências empíricas que apontavam o caráter protetor do apoio social em relação à hospitalização, recuperação de doenças, aposentadoria, estresse e depressão. Por sua vez, Cohen, Underwood e Gottlieb (2000) descrevem o apoio social como um processo que envolve interações com outras pessoas e que facilita o enfrentamento do estresse e outros estímulos aversivos.

Em um estudo de revisão, na mesma linha de raciocínio, Uchino e cols. (1996) encontraram evidências que as relações de suporte entre as pessoas protegem o indivíduo contra os perigos à saúde e dos eventos estressantes da vida. Fica claro nesse estudo que o suporte social pode proteger os indivíduos do agravamento de uma variedade de doenças, tais 
como artrite, tuberculose, depressão e alcoolismo. Além disso, os autores afirmam que a disponibilidade do suporte social pode, em contextos de adoecimento crônico, acelerar processos de recuperação e facilitar a adesão a tratamentos medicamentosos.

Entretanto, vale ressaltar que uma crítica freqüente às pesquisas sobre apoio social é a falta de consenso quanto à sua definição (Uchino, 2004). Tal dificuldade, segundo o autor, justifica-se pelo fato de que existem diversas áreas de pesquisa sobre o tema. Assim, é um conceito que tem sido investigado por antropólogos, epidemiologistas, enfermeiros, sociólogos, psicólogos, médicos, dentre outros profissionais. De forma abrangente e geral, o suporte social pode ser compreendido como qualquer processo em que relações sociais promovem saúde e bem-estar.

Atualmente, os estudos sobre suporte social focalizam dois aspectos em sua definição conceitual: estruturais ou funcionais (Seidl \& Tróccoli, 2006). Os autores classificam os aspectos estruturais como os relacionados à presença de apoio social independente da ocorrência de eventos estressores, valorizando questões como número de pessoas que integram a rede de apoio, por exemplo. $\mathrm{O}$ aspecto funcional diz respeito à disponibilidade e à modalidade de apoio recebido, assim como ao seu nível de satisfação, em relação a situações e contextos estressores específicos. Assim, um paciente pode receber apoio de muitas pessoas que provêm suporte social em geral, mas está insatisfeito e não tem disponibilidade de apoio em relação ao problema de saúde que o acomete, por exemplo.

$\mathrm{Na}$ área de saúde, o interesse pelo apoio social funcional tem sido relevante, tendo em vista a ocorrência de agravos que demandam apoio específico. Duas categorias têm predominado na literatura no que tange ao suporte funcional: instrumental e emocional (Seidl \& Tróccolli, 2006; Thoits, 1995). O suporte instrumental se refere à percepção e satisfação do indivíduo quanto à disponibilidade de apoio para questões operacionais relacionadas ao seu tratamento de saúde, atividades práticas do cotidiano, ajuda material ou financeira. $\mathrm{O}$ apoio emocional está relacionado à percepção e satisfação quanto à disponibilidade de escuta, atenção, informação, estima, companhia e apoio emocional em relação à condição de saúde e tratamento.
Pode-se afirmar que nos últimos 35 anos, diversas pesquisas buscaram conceituare operacionalizar $\mathrm{o}$ apoio social em termos estruturais e funcionais (Lett e cols., 2005). Tais trabalhos descrevem e analisam a percepção da pessoa a respeito do apoio recebido, dando um status psicológico aos estudos sobre suporte social.

\section{Suporte social, mortalidade e morbidade em doenças cardíacas}

Evidências apontam que existe uma relação direta entre interação social e mortalidade (Eng, Rimm, Fitzmaurice, \& Kawachi, 2002). Esses autores afirmam que, em geral, pessoas socialmente isoladas têm um risco aumentado de mortalidade em comparação com aquelas que mantêm relações com amigos, parentes e pessoas da comunidade. A maioria dos estudos sobre suporte social e progressão de doenças crônicas revela uma relação inversa entre as duas variáveis, ou seja, quanto maior e mais satisfatório o suporte social, mais lenta é a progressão da doença crônica (Cohen e cols., 2000). Os autores ressaltam que essa relação, na maioria das vezes, se refere a comportamentos que apresentam uma associação positiva com a melhora do quadro de saúde do doente crônico, como por exemplo, adesão ao tratamento e enfrentamento adequado.

Nesse contexto, Allen, Markovitz, Jacobs e Knox (2001) realizaram um estudo descritivo com 5115 participantes vinculados ao Coronary Artery Risk Development in Young Adults (CARDIA), que apresentavam escores elevados em uma medida de hostilidade. Os autores partiram do pressuposto que pacientes hostis apresentavam um perfil de adesão inadequado a comportamentos de saúde. Nesse cenário, foi investigado se havia associação entre nível mais elevado de suporte social e a adoção de comportamentos de saúde se comparada aos pacientes que apresentavam suporte social precário. O suporte social foi medido por meio de uma escala de onze itens. Comportamentos como consumo abusivo de álcool, não realização de atividades físicas e uso de tabaco foram examinados com a utilização de questionários semi-estrutrados. Os resultados confirmaram que o nível reduzido de suporte social esteve associado à baixa adesão a comportamentos de saúde que, por sua vez, relacionou-se diretamente à 
morbidade e mortalidade elevada desses pacientes. Dessa forma, é possível concluir que a disponibilidade de suporte social parece ter contribuído para a adesão a comportamentos promotores de saúde nos participantes do estudo.

Pesquisas documentam que há um índice elevado de morbidade e mortalidade em pacientes que estão socialmente isolados. Berkman, Glass, Brissete e Seeman (2000) realizaram um estudo prospectivo de nove anos em uma comunidade na qual o suporte social foi evidenciado com sendo determinante para o índice de mortalidade. Nessa população, o risco de mortalidade foi duas a três vezes maior em pessoas que careciam de apoio social do que nas que possuíam suporte social satisfatório. Resultados similares foram relatados no mesmo ano por Brummett e cols. (2001) em um estudo prospectivo de cinco anos que investigou as características de 590 pacientes coronarianos com sintomas depressivos. O objetivo principal do estudo foi verificar os tipos de pacientes que se beneficiariam do suporte social. Foi observado que aqueles que tinham menos recursos pessoais e sócio-ambientais de enfrentamento se beneficiaram mais do apoio social. Assim, pacientes que sofriam de depressão, eram idosos, tinham dificuldades financeiras e apresentavam grau elevado de gravidade da doença cardiovascular tinham risco duas vezes maior de morrer do coração se, além desses fatores, havia indisponibilidade ou inadequação do suporte social. Esses resultados indicam a influência relevante do apoio social, agravando a vulnerabilidade de pessoas que já apresentam outras condições de risco à saúde.

Uchino e cols. (1996) realizaram um estudo de revisão da literatura em que a relação entre suporte social em diversas enfermidades crônicas e processos fisiológicos referentes aos sistemas cardiovascular, endócrino e imunológico foram analisados. Os resultados revelaram a presença de uma associação significativa entre disponibilidade de suporte social e efeitos benéficos no funcionamento desses sistemas. Em 57 trabalhos analisados que investigaram apenas doenças do coração, a maioria evidenciou a ocorrência de associação positiva entre suporte social e melhora do funcionamento do sistema cardiovascular no que se refere à regulação da pressão arterial sistêmica e freqüência cardíaca.
Ao lado desses achados existem evidências consistentes de que o isolamento social e a falta de suporte são fatores de risco para o agravamento da doença coronariana (Angerer e cols., 2000; Wang, Mittleman, \& Orth-Gomer, 2005). Nesse contexto, Wang e cols. realizaram um estudo que teve como objetivo examinar a relação entre suporte social e a progressão de doença coronariana em 292 mulheres de meia idade que haviam sido internadas por infarto agudo do miocárdio ou angina instável. Três aspectos referentes às modalidades de apoio social foram estudados nessas pacientes com doença coronariana: suporte emocional, integração social e relações interpessoais. Foram usados dois instrumentos para acessar as variáveis do estudo. A progressão da doença cardiovascular foi avaliada por meio de uma arteriografia coronariana, que consiste em um exame computadorizado no qual as coronárias são visualizadas, assim como as mudanças que ocorrem no decorrer do tempo. Tais medidas foram verificadas aos três meses, seis meses e três anos após o episódio cardiovascular. Os resultados mostraram que houve progressão acelerada da doença coronariana em mulheres que relataram suporte social insuficiente $\mathrm{e}$ estavam em isolamento social, apresentando poucas relações interpessoais, enquanto que nas mulheres com bom nível de apoio o quadro clínico evoluiu mais lentamente. Um aspecto importante desse trabalho foi a utilização de instrumentos e medidas padronizadas e confiáveis, o que facilita a sua replicação em outras populações. Os autores concluíram que a inexistência ou precariedade do suporte social pode acarretar a progressão da doença coronariana.

Nessa mesma direção, Brummet e cols. (2001) investigaram 430 pacientes portadores de doença arterial coronariana e sua rede social de apoio. Por meio da aplicação de entrevistas semi-estruturadas e instrumentos validados, os autores verificaram a relação entre suporte social estrutural e risco de mortalidade cardíaca. Os resultados mostraram que a mortalidade foi maior entre os pacientes mais isolados: aqueles que tinham até três pessoas em sua rede de apoio apresentavam um risco relativo mais alto para mortalidade cardíaca, controlando-se variáveis como idade e gravidade da doença. As conclusões indicaram que pacientes com rede social de apoio numericamente insuficiente tinham risco mais elevado 
de mortalidade, sendo que esse não foi atribuído a outras variáveis como gravidade da doença, dados demográficos e estresse psicológico.

Lett e cols. (2007) realizaram um estudo com 2481 pacientes que sofreram infarto agudo do miocárdio e teve por objetivo comparar o impacto do suporte estrutural e de alguns aspectos do apoio funcional na mortalidade desses pacientes, por um período de quatro a cinco anos. Todos os pacientes responderam a quatro medidas de suporte social validadas. Os pacientes que receberam diagnóstico de depressão ou referiram pouco suporte social foram randomicamente alocados em dois grupos: de intervenção cognitivo-comportamental ou de cuidados médicos usuais. Inicialmente, não houve diferença no prognóstico dos pacientes dos dois grupos, entretanto, verificaram-se diferenças significativas na avaliação posterior. Assim, os resultados revelaram que pacientes com alto nível de suporte social percebido tiveram melhor prognóstico do que aqueles com baixo suporte.

Observa-se que a associação entre presença de suporte social e melhora do prognóstico do paciente cardíaco decorre do fato de que o apoio social funciona como moderador dos agravos secundários causados pela doença coronariana, como depressão (Barefoot e cols., 2000), estresse pós-traumático e ansiedade (Pedersen, Middel, \& Larsen, 2002). Nessa perspectiva, a satisfação com a presença de apoio social é forte preditor de uma melhor reabilitação e, consequientemente, de um melhor funcionamento físico, psicológico e cognitivo do paciente (Shen, McCreary, \& Myers, 2004).

Em suma, conclui-se, de acordo com as evidências obtidas nesses trabalhos, que o suporte social parece influenciar a morbi-mortalidade por doenças coronarianas, podendo exercer influência em estágios específicos desses quadros clínicos. Entretanto, se o apoio social prediz o desenvolvimento e a incidência do quadro coronariano, não há evidências suficientes. Um número maior de estudos com delineamento longitudinal é necessário para examinar a função que o suporte social exerce por um período de tempo prolongado. Além disso, esses estudos deveriam analisar múltiplas variáveis relacionadas ao suporte social e à doença coronariana.

\section{Suporte social e adesão a comportamentos de saúde}

Pesquisadores têm procurado verificar a relação existente entre disponibilidade e satisfação com o apoio social e melhoria do quadro clínico, não evolução da doença e adesão a comportamentos de saúde. Segundo Straub (2005), a adesão ao tratamento refere-se à disposição do paciente em adotar e seguir o regime de tratamento recomendado e conseguir fazê-lo. Nesse contexto, o suporte social parece influenciar positivamente a adesão à prática de atividade física, adoção de alimentação adequada, cessação do tabagismo e redução do consumo de álcool (Eng e cols., 2002). Wallace, Buckwort, Kirby e Sherman (2000) demonstraram que o suporte de amigos e familiares prediz a participação em atividades físicas e, conseqüentemente, leva à diminuição do sedentarismo em pacientes coronarianos. Em suma, a literatura aponta que o fornecimento de suporte social contribui para a melhora das condições de saúde dos pacientes, uma vez que tende a aumentar a adesão ao tratamento.

Essa relação é ilustrada por Franks e cols. (2006) em um estudo no qual examinaram o comportamento de 94 esposas de pacientes em reabilitação cardíaca pós-infarto, por meio de uma escala Likert ( $0=$ nunca forneço; $4=$ sempre forneço) desenvolvida para mensurar o envolvimento da parceira na adesão do paciente a comportamentos de saúde. O apoio social relacionado a essas condutas foi definido como "comportamentos de assistência e reforço aos esforços do paciente em iniciar e manter mudanças de hábitos de saúde prescritos” (p.313). Os resultados mostraram uma relação positiva entre disponibilidade de apoio e aumento da adesão a comportamentos de saúde dos pacientes.

Nesse mesmo contexto, Boutin-Foster (2005a) afirma que pacientes com falta de suporte adequado parecem apresentar maiores dificuldades para aderir a comportamentos que promovam a saúde e o bemestar (atividade física regular, alimentação adequada e cessação do hábito de fumar), tendendo à piora de suas condições físicas. Nessa perspectiva, a autora investigou as modalidades de suporte social instrumental percebido por pacientes coronarianos como mais adequados para a modificação de comportamentos 
de saúde. Uma amostra composta de 63 pessoas participou desse estudo qualitativo. Os pacientes descreveram mudanças realizadas no estilo de vida a fim de se manterem saudáveis, bem como os tipos de suporte instrumental fornecidos por pessoas da rede social. Os resultados mostraram que as mudanças no estilo de vida mais relatadas foram modificações na dieta, diminuição das atividades diárias rotineiras, aumento da frequiência às consultas médicas, ingestão correta de medicações e adesão à atividade física. Os tipos de suporte social instrumental que foram referidos como sendo os que mais ajudaram à realização de tais mudanças foram aqueles que: (1) facilitaram e tornaram mais prático o engajamento em tais comportamentos; (2) aliviaram situações estressantes; (3) facilitaram o processo de recebimento de cuidados médicos.

Por fim, a literatura aponta que a intervenção direcionada ao fornecimento de suporte social adequado, desenvolvida com cuidadores de pessoas acometidas por enfermidades cardíacas, pode contribuir para a melhora das condições de saúde dos pacientes, uma vez que tende a aumentar a adesão ao tratamento e seu engajamento em comportamentos de saúde.

\section{A importância da orientação aos cuidadores para o fornecimento de apoio}

As intervenções para promover o apoio social têm se mostrado eficazes no tratamento de diversas doenças quando comparadas apenas a cuidados médicos usuais (Hogan, Linden, \& Najarian, 2002). Esses autores, em um trabalho de revisão com 100 artigos sobre diferentes intervenções em suporte social, mostraram que em $83 \%$ dos estudos tais intervenções revelaram resultados benéficos em domínios como bem-estar, aumento da rede de apoio social e/ ou melhora do quadro de saúde do paciente.

Martire e Schulz (2007), em um estudo empírico, fizeram uma comparação entre disponibilidade de cuidados médicos com orientação familiar, de cuidados médicos com orientação somente ao paciente e de cuidados médicos exclusivos, sem nenhum tipo de ação educativa. Resultados indicaram que, tanto a ação que incluiu a intervenção familiar quanto aquela que abarcou orientação dada aos pacientes, foram mais eficazes do que os cuidados médicos exclusivos. A eficácia da intervenção foi medida a partir da avaliação de indicadores acerca da melhora do quadro de saúde dos pacientes. Concluiu-se que é de suma importância, assim como a orientação aos pacientes, a realização de estudos com intervenções familiares que incluam conteúdos que objetivam sensibilizar e orientar os familiares no fornecimento de suporte social adequado aos pacientes para que os mesmos vivam de forma mais independente e autônoma possível. Tais intervenções parecem ter eficácia maior do que a disponibilização de orientações dadas apenas aos pacientes ou de cuidados médicos exclusivos.

Entretanto, como enfatizado por diversos autores, no contexto da doença coronariana existe uma disposição de amigos e membros da família no sentido de fornecer suporte social. Apesar de ser reconhecido como benéfico, há evidências sugerindo que essa iniciativa pode ser percebida pelo paciente como problemática e causar prejuízos à sua saúde (Itkowitz, Kerns, \& Otis, 2003). Boutin-Foster (2005b) verificou esse fato em um estudo qualitativo com 59 pessoas internadas devido ao diagnóstico de síndrome coronariana aguda. A pesquisa buscou identificar as características problemáticas do suporte social a partir da perspectiva do paciente. Foi feita a seguinte pergunta aos participantes: "Você poderia descrever as coisas que membros de sua família, amigos próximos e profissionais da saúde fizeram durante esse período tentando fornecer suporte e ajuda, e que você sentiu que não ajudou ou que lhe causou mais estresse?" (Boutin-Foster, 2005b, p. 2). Os resultados indicaram que muitas ações realizadas pelo provedor do apoio social, que tinham a intenção de serem positivas, foram percebidas como problemáticas pelos pacientes, consideradas excessivas, incongruentes com as suas necessidades, chegando a gerar sentimentos negativos.

O fornecimento de orientação ao cuidador sobre as peculiaridades do quadro de saúde do paciente, assim como sobre as formas de fornecer apoio social instrumental e emocional adequados, é fundamental para promover o desenvolvimento de suporte relevante e, conseqüentemente, auxiliar o processo de reabilitação e promoção da saúde do paciente. 


\section{Considerações finais}

É evidente que o provimento de cuidados de saúde de qualidade ao paciente coronariano será uma das grandes preocupações dos profissionais do século XXI. Nesse contexto, a compreensão sobre como a enfermidade afeta a rede social do paciente será de grande importância para o entendimento acerca da evolução do seu quadro clínico. Entretanto, é surpreendente o número limitado de pesquisas que abordam o tema da relação entre cuidador e paciente coronariano. Assim sendo, faz-se necessário o desenvolvimento de estudos com propostas de intervenção, que investiguem o papel de cuidadores na disponibilidade de suporte social que favoreça a eficácia do tratamento de pacientes acometidos por enfermidade coronariana.

O principal objetivo desse artigo foi examinar evidências que relacionam o suporte social a diferentes aspectos da doença coronariana. Percebe-se, nessa breve revisão, que existem fortes evidências da relação positiva entre disponibilidade de suporte social e melhora da saúde do paciente coronariano.

Fazendo uma análise metodológica dos estudos que investigaram suporte social e doenças coronarianas incluídos nessa revisão, percebe-se que a maioria utilizou-se de delineamentos transversais, sendo que poucos lançaram mão de metodologia experimental ou quase experimental. Esse fato, não obstante as implicações positivas para justificar a relação existente entre suporte social, progressão e mortalidade da doença coronariana, limitam a verificação de eventuais relações causais entre as variáveis estudadas. A generalização dos resultados também fica comprometida devido ao controle precário das variáveis. Faz-se necessário pesquisas de cunho experimental com maior rigor no controle das variáveis analisadas.

Quanto ao tema suporte social e cardiologia, percebe-se que essa linha de investigação é fundamental, visto que muitos pacientes carecem de suporte adequado. O mais importante é que muitas vezes as fontes de suporte - pessoas da rede sócio-familiar do paciente - não fornecem apoio adequado por falta de conhecimento ou de informação sobre as condutas que poderiam adotar. Ademais, a divulgação de resultados sobre a relação exis- tente entre fornecimento de suporte adequado e melhoria da saúde do paciente seria uma forma de promover qualidade de vida.

\section{Referências}

Allen, J., Markovitz, J., Jacobs, R. D., \& Knox, S. S. (2001). Social support and health behavior in hostile blacks and white men and women in CARDIA. Psychosomatic Medicine, 63, 609-618.

Angerer, P., Siebert, U., Kothny, W., Muhlbauer, D., Mudra, H., \& Schacky, C. (2000). Impact of social support, clinical hostility and anger expression on progression of coronary artherosclerosis. Journal of the American College of Cardiology, 36, 1781-1788.

Araújo, S. S. C., Freire, D. B. L., Padilha, D. M. P., \& Baldisseroto, J. (2006). Suporte social, promoção de saúde bucal da população idosa no Brasil. Interface- Comunicação, Saúde e Educação, 10, 203-216.

Barefoot, C. J., Brummett, H. B., Clapp-Channing, E. N., Siegler, C. I., Vitalino, P. P., Williams, B. R., \& Mark, B. D. (2000). Moderators of the effect of social support on depressive symptoms in cardiac patients. American Journal of Cardiology, 86, 438-442.

Beltrão, M. R. L. R., Vasconcelos, M. G. L., Pontes, C. L., \& Albuquerque, M. C. (2007). Câncer infantil: Percepções maternas e estratégias de enfrentamento frente ao diagnóstico. Jornal de Pediatria, 83, 562-566.

Berkman, L. F., Glass, T., Brissete I., \& Seeman, T. E. (2000). From social integration to health: Durkheim in the new millennium. Social Science Medicine, 51, 843- 857.

Boutin-Foster, C. (2005a). Getting to the heart of social support: A qualitative analysis of the types of instrumental support that are most helpful in motivating cardiac risk factor modification. Heart \& Lung, 34(1), 22-29.

Boutin-Foster, C. (2005b). In spite of good intentions: Patients perspectives on problematic social support interactions. Health and Quality of Life Outcomes, 52(3), 1-7. 
Brasil. Ministério da Saúde. Indicadores e dados básicos - Brasil 2006. Recuperado em 11 março 2008, de http://tabnet.datasus.gov.br/cgi/idb2006/ matriz.htm.

Brummett, B., Barefoot, J., Siegler, I., Clapp-Channing, N., Lytle, B., Bosworth, H. Williamns, R., \& Mark, D. (2001). Characteristics of socially isolated patients with coronary artery disease who are at elevated risk of mortality. Psychosomatic Medicine, 63, 267-272.

Cobb, S. (1976). Social support as a moderator of life stress. Psychosomatic Medicine,38, 300-314.

Cohen, S., Underwood, L. G., \& Gottlieb, H. B. (2000). Social support measurement and intervention. New York: Oxford University Press.

Cupertino, A. P. F. B., Oliveira, B. H. D., Guedes, D. V., Coelho, E. R., Milano, R. S., \& Rubac, J. S. (2006). Estresse e suporte social na infância e adolescência relacionados com sintomas depressivos em idosos. Psicologia Reflexão Crítica, 19, 371-378.

Eng, M. P., Rimm, B. E., Fitzmaurice, G., \& Kawachi, I. (2002). Social ties and change in social ties to subsequent total and cause-specific mortality in coronary heart. Heart disease incidence in men. American Journal of Epidemiology, 155, 700-709.

Franks, M., Rook, K., Keteyian, S., Stephens, M., Franklin, B., \& Artinian, N. (2006). Spouses' provision of health-related support and control to patients participating in cardiac rehabilitation. Journal of Family Psychology, 20 , 311-318.

Giannotti, A. (2002). Prevenção da doença coronariana: Perspectiva psicológica em um programa multiprofissional. Psicologia USP, 13(1),167-195.

Hogan, B., Linden, W., \& Najarian, B. (2002). Social support interventions. Do they work? Clinical Psychology Review, 22, 381-440.

Itkowitz, N., Kerns, R., \& Otis, J. (2003). Support and coronary heart disease. The importance of significant other response. Journal of Behavioral Medicine, 26, 19-30.
Lett, H., Blumenthal, J., Babyak, M., Strauman,T., Robins, C., \& Sherwoo, A. (2005). Social support and coronary heart disease: Epidemiologic evidence and implications for treatment. Psychosomatic Medicine, 67, 869-878.

Lett, H., Blumenthal, J., Carney, R., Burg, M., Jaffe, A., Catellier, D., Beckman, L., Mitchell, P., \& Schneiderman, N. (2007). Social support and prognosis in patients at increased psychosocial risk recovering from myocardial infarction. Health Psychology 26, 418-427.

Mansur, A. P., Armaganijan, D., Amino, J. G., Sousa, A. C., Simão, A. F., Brito, A. X. e cols. (2004). Diretrizes de doença coronariana crônica angina estável. Arquivos Brasileiros de Cardiologia, 83(Supl. 2), 2-43.

Martire, M. L., \& Schulz, R. (2007). Involving family in psychosocial interventions for chronic illness. Current Directions in Psychological Science, 16, 90-94.

Matsukura, T. S., Marturano, E. M., \& Oishi, J. (2002). O Questionário de Suporte Social (SSQ): Estudos da adaptação para o português. Revista Latino-Americana de Enfermagem, 10, 675-681.

Pedersen, S., Middel, B., \& Larsen, M. (2002). The role of personality variables and social support in distress and perceived health in patients following myocardial infarction. Journal of Psychosomatic Research, 53, 1171-1175.

Pinto, J. L. G., Garcia, A. C. O., Bocchi, S. C. M., \& Carvalhaes, M. A. B. L. (2006). Característica do apoio social oferecido a idosos na área rural assistida pelo PSF. Ciência e Saúde Coletiva, $11,753-764$.

Seidl, E. M. F., \& Tróccoli, B. T. (2006). Desenvolvimento de escala para avaliação do suporte social em pessoas vivendo com HIV/aids. Psicologia Teoria e Pesquisa, 22, 317-326.

Shen, B., McCreary. P. C., \& Myers, F. H. (2004). Independent and mediated contributions of personality, coping, social support and depressive symptoms to physical functioning outcome among patients. Journal of Behavioral Medicine, 27, 39-61. 
Straub. O. R. (2005). Psicologia da saúde. Porto Alegre: Artmed.

Thoits, P. A. (1995). Stress, coping and social support processes: Where are we? What next? [Special issue]. Journal of Health and Social Behavior, 53-79.

Uchino, N. B. (2004). Social support and physical health. New Haven: Yale University Press.

Uchino, N. B., Cacioppo, T. J., \& Kiecolt-Glaser, K. J. (1996). The relationship between social support and physiological processes: A review with emphasis on underlying mechanisms and implications for health. Psychological Bulletin, 119, 488-531.

Wallace, L. S., Buckwort, J., Kirby, T. E., \& Sherman, W. N. (2000). Characteristics of exercise behavior among students: Application of social cognitive theory to predict stage change. Preventive Medicine, 31, 494-505.

Wang, H., Mittleman, A. M., \& Orth-Gomer, K. (2005). Influence of social support on progression of coronary artery disease in women. Social Science and Medicine, 60, 599-607.

World Health Organization (s.d.). Death and daly estimates for 2002 by cause for WHO member states. Recuperado em 21 janeiro 2008, de http:// www.who.int/healthinfo/bod/en/index.html.

Artigo recebido em 29/10/2007.

Aceito para publicação em 25/07/2008.

Este trabalho é parte da dissertação de mestrado realizada pela primeira autora, sob orientação da segunda autora, com apoio financeiro da CAPES.

Endereço para correspondência:

Profa. Dra. Eliane Maria Fleury Seidl. Universidade de Brasília. Instituto de Psicologia. Campus Universitário Darcy Ribeiro. CEP 70910-900. BrasíliaDF, Brasil.
Marcela Abreu-Rodrigues é Mestre em Psicologia pelo Instituto de Psicologia da Universidade de Brasília.

Eliane Maria Fleury Seidl é Professor Adjunto do Instituto de Psicologia da Universidade de Brasília. 